\title{
Optimum structure for a uniform load over multiple spans
}

\author{
Aleksey V. Pichugin ${ }^{1} \cdot$ Andrew Tyas $^{2} \cdot$ Matthew Gilbert ${ }^{2} \cdot$ Linwei He $^{2}$
}

Received: 10 September 2014 / Revised: 26 May 2015 / Accepted: 2 June 2015 / Published online: 10 July 2015

(C) Springer-Verlag Berlin Heidelberg 2015

\begin{abstract}
This paper presents a new half-plane Michell structure that transmits a uniformly distributed load of infinite horizontal extent to a series of equally-spaced pinned supports. A full kinematic description of the structure is obtained for the case when the maximum allowable tensile stress is greater than or equal to the allowable compressive stress. Although formal proof of optimality of the solution presented is not yet available, the proposed analytical solution is supported by substantial numerical evidence, involving the solution of problems with in excess of 10 billion potential members. Furthermore, numerical solu-
\end{abstract}

This is an extended version of a paper prepared by Pichugin et al. (2011) for the 9th World Congress on Structural and Multidisciplinary Optimization, subsequently awarded the 2011 ISSMO/Springer Prize.

Electronic supplementary material The online version of this article (doi:10.1007/s00158-015-1278-0) contains supplementary material, which is available to authorized users.

Aleksey V. Pichugin

aleksey.pichugin@brunel.ac.uk

Andrew Tyas

a.tyas@sheffield.ac.uk

Matthew Gilbert

m.gilbert@sheffield.ac.uk

Linwei He

cip111h@sheffield.ac.uk

1 Department of Mathematics, CEDPS, Brunel University, Uxbridge UB8 3PH, UK

2 Department of Civil and Structural Engineering, University of Sheffield, Sheffield, S1 3JD, UK tions for various combinations of unequal allowable stresses suggest the existence of a family of related, simple, and practically relevant structures, which range in form from a Hemp-type arch with vertical hangers to a structure which strongly resembles a cable-stayed bridge.

Keywords Plastic design · Truss optimization · Michell structure $\cdot$ Uniformly distributed load

\section{Introduction}

In his groundbreaking contribution to the field of structural optimization, Michell (1904) formulated the sufficient conditions for a truss with equal tensile and compressive yield stresses to have the least volume. Hemp (1973) showed that these conditions are also necessary, and generalised them to the case of unequal allowable stresses; see the re-evaluation and clarification of these results by Rozvany (1996). In trusses satisfying the Michell-Hemp criteria, the magnitudes of the tensile and/or compressive stresses in load-carrying members must everywhere be at maximum allowable values, and the virtual strains in such members must not exceed these limiting values. The corresponding displacement field must remain continuous throughout the design domain and satisfy the kinematic restrictions imposed on the solution.

The Michell-Hemp criteria can be satisfied in several different ways, implying that every domain containing an optimal structure can be split into one or more regions, distinguished by values of the member force components $f^{\prime}$ and $f^{\prime \prime}$ and associated principal strains $\varepsilon^{\prime}$ and $\varepsilon^{\prime \prime}$ :

$$
\begin{gathered}
T: f^{\prime}<0, f^{\prime \prime}>0, \varepsilon^{\prime}=-\ell \sigma / \sigma_{C}, \varepsilon^{\prime \prime}=\ell \sigma / \sigma_{T} ; \\
S^{C}: f^{\prime}<0, f^{\prime \prime}<0, \varepsilon^{\prime}=-\ell \sigma / \sigma_{C}, \varepsilon^{\prime \prime}=-\ell \sigma / \sigma_{C} ;
\end{gathered}
$$




$$
\begin{aligned}
S^{T}: f^{\prime}>0, f^{\prime \prime}>0, \varepsilon^{\prime}=\ell \sigma / \sigma_{T}, \varepsilon^{\prime \prime}=\ell \sigma / \sigma_{T} & \\
R^{C}: f^{\prime}= & 0, f^{\prime \prime}<0, \\
& -\ell \sigma / \sigma_{C} \leq \varepsilon^{\prime} \leq \ell \sigma / \sigma_{T}, \varepsilon^{\prime \prime}=-\ell \sigma / \sigma_{C} ; \\
R^{T}: f^{\prime}>0, f^{\prime \prime} & =0, \\
\varepsilon^{\prime} & =\ell \sigma / \sigma_{T},-\ell \sigma / \sigma_{C} \leq \varepsilon^{\prime \prime} \leq \ell \sigma / \sigma_{T} .
\end{aligned}
$$

Within (1)-(5), $\sigma_{T}$ and $\sigma_{C}$ denote the maximum allowable tensile and compressive stresses, $\sigma=\left(\sigma_{T}+\sigma_{C}\right) / 2$ and $\ell$ is a positive infinitesimal. Optimal trusses may also contain regions of uniform tension and/or compression, see Rozvany et al. (1995). All trusses constructed by Michell (1904), as well as the majority of optimal trusses identified in the early literature, only feature one or several regions of type $T$; thus, the term 'Michell structure' is sometimes considered to be synonymous with definition (1). In our paper we use this term in a more general way, to describe any structure that satisfies the Michell-Hemp criteria, which implies that any number of regions (1)-(5) can be present.

The deceptive simplicity of the specified criteria should not obscure the fact that there is no known procedure for verifying whether a Michell structure exists for a given problem specification, or for determining its form. Unsurprisingly, the number of Michell structures to have been identified to date is not large, see e.g. Michell (1904), Chan (1962), Chan (1967), Hemp (1973), Lewiński et al. (1994), and Rozvany (1998). Furthermore, whilst some notable exceptions exist, see Hemp (1974), Chan (1975), Sokół and Lewiński (2010), and Tyas et al. (2011), the majority of known Michell structures are designed to support only a single external point load.

In this paper, we present details of an apparently new Michell structure, for a problem which appears to have been hitherto overlooked. The problem involves a uniformly distributed vertical load applied along a horizontal line spanning across an infinite number of equally-spaced pinned supports. The motivation for this configuration originates from the (still unsolved) classical problem of finding the optimal half-plane structure to transmit a uniformly distributed load along a line between two level pinned supports, to these supports, see Hemp (1974) and Chan (1975). In the case of equal allowable stresses and an infinite number of equally-spaced supports, the resulting geometry of the Michell structure, and the mathematical solution for kinematic fields, all turn out to be comparatively simple. Importantly, the volume per single span of the resulting structure is approximately $11.0 \%$ lower than that of the parabolic arch with vertical hangers and $7.86 \%$ lower than that of the classical solution by Hemp (1974), which is known to be sub-optimal. We stop short of proving the optimality of the proposed structure for the half-plane; however, results from numerical simulations presented in the paper appear to support our claim. We also present a number of numerical solutions for similar problems with unequal allowable stresses, suggesting that a wider family of related, simple and practically relevant structures exists.

\section{An auxiliary problem}

Before analysing our main problem, featuring an infinite number of equally-spaced supports, it is instructive to examine a simpler configuration. Consider a uniformly distributed load ( $w$ per unit length) that is applied to a horizontal line segment of length $2 L$, and needs to be transmitted to a pinned support at the centre of the segment. It is not difficult to verify that the suitable optimal solution for the upper half-plane is a 'half-wheel', the structure comprising concentric semicircles and orthogonal radii, as shown on Fig. 1. A nearly identical structure has been constructed for the problem involving external vertical point loads at points $x= \pm L$ by Michell (1904) and Hemp (1973). The outer rim of their classic solution represents a concentrated member, connected to the support by the continuum of straight spokes. The solution for the distributed load, described in this section, does not have concentrated members and would be more correctly interpreted as a 'half-disc', a mesh of mutually orthogonal radial and concentric members.

The structure is conveniently mapped by the orthogonal curvilinear system $(\alpha, \beta)$, such that

$\alpha=r, \quad \beta=\theta, \quad \phi=\beta+\frac{\pi}{2}, \quad A=1, \quad B=\alpha$,

in which $r, 0 \leqslant r \leqslant L$, is the linear distance from the support, $\theta,-\pi / 2 \leqslant \theta \leqslant \pi / 2$, the polar angle measured

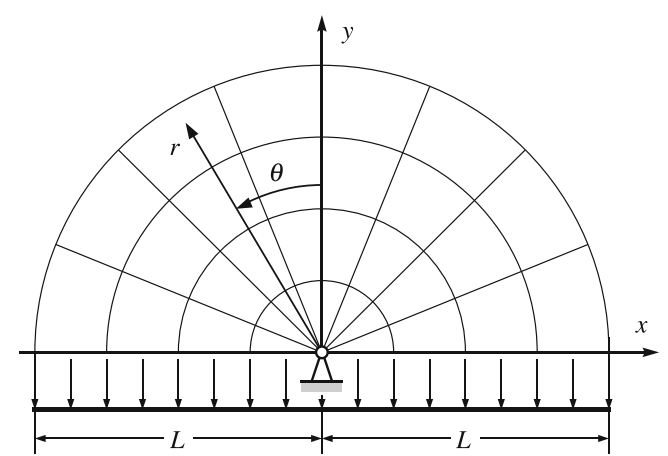

Fig. 1 The Michell half-wheel transmitting a uniformly distributed load to a single pinned support 
counter-clockwise from the vertical symmetry axis and $\phi$ the angle measured from the horizontal line to the tangent of an $\alpha$-line. Functions $A$ and $B$ are the Lamé coefficients, i.e. the scale factors for the chosen system of orthogonal curvilinear coordinates (Morse and Feshbach 1953). A suitable strain field is given by

$u=-\ell \sigma \frac{\alpha}{\sigma_{C}}, \quad v=\ell \sigma \frac{\alpha \beta}{\sigma_{*}}, \quad \omega=\ell \sigma \frac{\beta}{\sigma_{*}}$,

where $u$ and $v$ denote displacements along $\alpha$ and $\beta$, respectively, $\omega$ denotes the rotation and $\sigma_{*}=\sigma_{C} \sigma_{T} /\left(\sigma_{C}+\sigma_{T}\right)$. If $T^{\prime}$ and $T^{\prime \prime}$ denote the end loads per unit coordinate difference in the $\alpha$ and $\beta$ directions, then they must satisfy the standard equilibrium equations in curvilinear coordinates:

$\frac{\partial T^{\prime}}{\partial \alpha}=T^{\prime \prime} \frac{\partial \phi}{\partial \beta}, \quad \frac{\partial T^{\prime \prime}}{\partial \beta}=-T^{\prime} \frac{\partial \phi}{\partial \alpha}$,

see Hemp (1973). In our case $\partial T^{\prime \prime} / \partial \beta=0$, and the equilibrium of vertical components of forces acting along the bottom of the structure requires that $T^{\prime \prime}=w$. Equation $(8)_{1}$ can now be integrated, yielding $T^{\prime}=w \alpha+t^{\prime}(\beta)$. One needs to add another boundary condition to fully specify the force field within the structure. For example, if $T^{\prime}$ is required to vanish along the outer rim of the structure, then $t^{\prime}(\beta)=-w L$ and $T^{\prime}=w(\alpha-L)$, hence, completing the solution.

The volume of the resulting structure is found from the virtual work of external loads, which in our case yields

$W_{\text {aux }}=\frac{2}{\ell \sigma} \int_{0}^{L}-\left.w v\right|_{\beta=-\pi / 2} \mathrm{~d} \alpha=\frac{\pi}{2} \frac{w L^{2}}{\sigma_{*}}$.

Strain field (7) may be trivially extended to cover the entire half-plane, thus signalling the global optimality of the solution. Although not a structure in the conventional sense, this solution for a single pinned support still has lower volume than, the admittedly suboptimal, half-plane structure constructed by Hemp (1974) for the case of two pinned supports. Indeed, when $\sigma_{C}=\sigma_{T}$, (9) reduces to $W_{\text {aux }}=\pi w L^{2} / \sigma$, whereas the volume of Hemp's structure is $3.155 w L^{2} / \sigma$.

\section{The virtual displacement field}

Perhaps unsurprisingly, the strain field described in Section 2 cannot be immediately adopted to problems featuring multiple supports. Indeed, if we were to consider two level supports, and attempt to match two copies of field (7), expanding from each of the supports, then this would be found to be impossible due to the monotonic variation of each local $u$ and $v$ as functions of local $\alpha$. Motivated by this observation, we consider an extension of the structure from

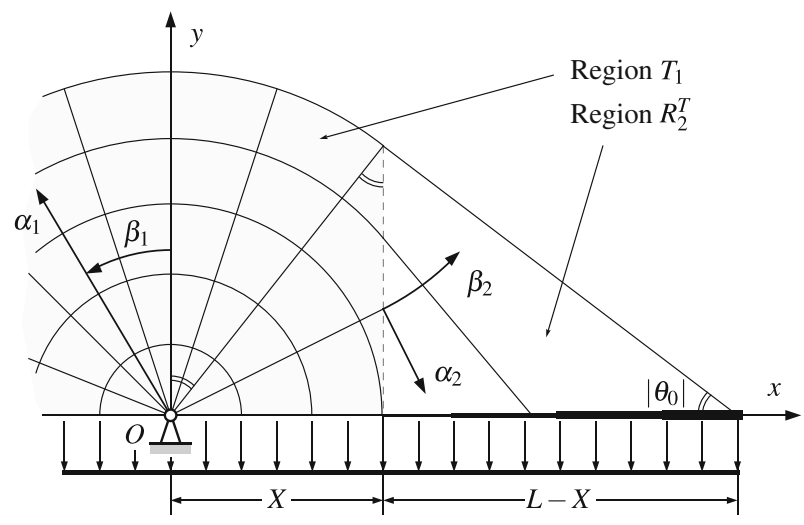

Fig. 2 Half-span of the proposed structure

Section 2, with a half-span as shown in Fig. 2 (we assume that the other half is obtained by reflecting the structure about the vertical $O y$ ). The original quarter-wheel of width $L$ is cut along the vertical line originating from the point $x=X$ in the global Cartesian coordinate system $O x y$. We assume that the virtual displacement field within the shaded region $0 \leqslant x \leqslant X$, now denoted as $T_{1}$, is defined precisely the same as described in Section 2. Outside $T_{1}$, the trajectories of tensile circumferential members are continued as straight ties until they reach $y=0$. We denote this second region as $R_{2}^{T}$, in accordance with definition (5). In order to ensure that the inclined ties equilibrate the vertical distributed load, one has to complete the construction by adding a horizontal concentrated member along the bottom of $R_{2}^{T}$; this member is best interpreted as a degenerate region $S_{3}^{T}$, see definition (3).

Parameter $X$, presently unknown, fully determines the geometry of the optimal structure. The additional degree of freedom, offered by having $X$ within our formulation, will turn out to be sufficient to ensure that the horizontal displacement vanishes at $x=L$. This will enable us to reflect the structure about the vertical $x=L$ and, consequently, by copying and reflecting the field, to construct the virtual displacement field satisfying all kinematic requirements, i.e. vanishing displacement at the infinite sequence of equally-spaced level pinned supports. Simple geometric considerations lead to the conclusion that $X$ can also be written in terms of the angle $\theta_{0}$ between axis $O x$ and the top tie within region $R_{2}^{T}$ :

$X=L \sin ^{2} \theta_{0}$.

Throughout this paper we are using right-handed coordinate systems, with positive angles measured counter-clockwise. Because of this, rather than working with an obtuse angle, 
it will be more convenient to use negative angle $\theta_{0}$. In view of relationship (10), we shall be using parameters $X$ and $\theta_{0}$ concurrently as an additional way of simplifying some of the forthcoming expressions.

The mathematical description of this structure calls for the use of several curvilinear coordinate systems. The region $T_{1}$ can be fully described using the same coordinate system as in Section 2. Thus, we assume that $\alpha_{1}, \beta_{1}, \phi_{1}, A_{1}, B_{1}$, $u_{1}, v_{1}$ and $\omega_{1}$ are defined precisely as in (6), (7) (note that here and henceforth the numeric subscripts indicate which specific region a given quantity relates to). The only difference concerns ranges of the variation of the coordinates. Since the verticals $x= \pm X$ are described within $T_{1}$ by equation $\alpha_{1}=-X \sin \beta_{1}$, therefore, $-\pi / 2 \leqslant \beta_{1} \leqslant \pi / 2$ (as before) and $0 \leqslant \alpha_{1} \leqslant \min \left\{L\left|\sin \theta_{0}\right|, X /\left|\sin \beta_{1}\right|\right\}$. In particular, along the boundary with region $R_{2}^{T}$, the curvilinear displacements and rotation are given by

$$
\begin{aligned}
& \left.u_{1}\right|_{x=X}=\frac{\ell \sigma X}{\sigma_{C} \sin \beta_{1}},\left.\quad v_{1}\right|_{x=X}=-\frac{\ell \sigma X \beta_{1}}{\sigma_{*} \sin \beta_{1}}, \\
& \left.\omega_{1}\right|_{x=X}=\frac{\ell \sigma \beta_{1}}{\sigma_{*}} .
\end{aligned}
$$

The curvilinear coordinate system appropriate for describing the strain field within region $R_{2}^{T}$ is harder to formulate. The systems of straight, non-intersecting ties associated with regions described by (5) may be analysed using the mathematical formalism by Hemp (1973, Section 4.2). Since line $x=X$ is the boundary between $T$ and $R$ regions, we know that ties within $R_{2}^{T}$ must be orthogonal to the struts within $T_{1}$. The gradient of each tie within $R_{2}^{T}$ is given by the value of $\tan \beta_{1}$ at $x=X$, where the tie "peels off" the fan. Thus, it is convenient to introduce coordinate $\beta_{2}$ within region $R_{2}^{T}$ to be the same angle as $\beta_{1}$, e.g., the bottom left corner of $R_{2}^{T}$ corresponds to $\beta_{2}=-\pi / 2$, whereas the uppermost tie corresponds to $\beta_{2}=\theta_{0}$. All ties within $R_{2}^{T}$ can be described as the family of straight lines parametrised by $\beta_{2}$ :

$\Phi\left(x, y, \beta_{2}\right)=x-y \cot \beta_{2}-X\left(1+\cot ^{2} \beta_{2}\right)=0$.
These lines envelop an evolute, the equation of which can be found by solving simultaneously $\Phi\left(x, y, \beta_{2}\right)=0$ and $\partial \Phi\left(x, y, \beta_{2}\right) / \partial \beta_{2}=0$, which yields

$y^{2}+4 X x-4 X^{2}=0$.

In an orthogonal coordinate system with $\alpha_{2}$ defined as the distance from a fixed involute, (14) may be alternatively written as $\alpha_{2}+F\left(\beta_{2}\right)=0$. Here $F\left(\beta_{2}\right)$ is the arc length measured along the evolute from the point where $\alpha_{2}=0$. Since evolute (14) touches the bottom left corner of $R_{2}^{T}$, it is convenient to use the involute passing through this point as the coordinate axis. We can now integrate along the evolute to obtain the full description of our curvilinear coordinates in the form

$\phi_{2}=\beta_{2}+\frac{\pi}{2}, \quad A_{2}=1, \quad B_{2}=\alpha_{2}+F\left(\beta_{2}\right)$,

where

$F\left(\beta_{2}\right)=X\left(\cot \beta_{2} \csc \beta_{2}-\ln \left[\cot \beta_{2}-\csc \beta_{2}\right]\right)$,

see also Hemp (1973). The Cartesian description of coordinate lines in $\left(\alpha_{2}, \beta_{2}\right)$ is obtained by computing

$x+\mathrm{i} y=X+\alpha_{2} \mathrm{e}^{\mathrm{i} \beta_{2}}+\mathrm{i} \int_{-\pi / 2}^{\beta_{2}} \mathrm{e}^{\mathrm{i} \xi} F(\xi) \mathrm{d} \xi$,

which leads to the explicit formulae

$x=\left(\alpha_{2}-X \ln \left[\cot \beta_{2}-\csc \beta_{2}\right]\right) \cos \beta_{2}+X$,

$y=\left(\alpha_{2}-X \ln \left[\cot \beta_{2}-\csc \beta_{2}\right]\right) \sin \beta_{2}-X \cot \beta_{2}$.

An additional test of the validity of these equations may be performed by directly computing the Lamé parameters from (18) and (19). The resulting expressions match (15) exactly. Table 1 presents some useful relationships between coordinates of various lines and points within the global Cartesian and the local curvilinear coordinate systems.

Given orthogonal coordinates (15), we can formulate the system of partial differential equations describing principal and shear strains, as well as the rotation, in the form:

$$
\begin{aligned}
& \frac{\partial u_{2}}{\partial \alpha_{2}}=\frac{\ell \sigma}{\sigma_{T}}, \quad v_{2}=\omega_{2}\left(\alpha_{2}+F\left(\beta_{2}\right)\right)+\frac{\partial u_{2}}{\partial \beta_{2}}, \\
& \omega_{2}=\frac{\partial v_{2}}{\partial \alpha_{2}}, \quad \varepsilon_{2}^{\prime \prime}=\left(\alpha_{2}+F\left(\beta_{2}\right)\right)^{-1}\left(\frac{\partial v_{2}}{\partial \beta_{2}}+u_{2}\right) .
\end{aligned}
$$

Table 1 Significant lines and points within the coordinate system $\left(\alpha_{2}, \beta_{2}\right)$

\begin{tabular}{lll}
\hline Cartesian & Curvilinear & Significance \\
\hline$x=X$ & $\alpha_{2}=X \ln \left[\cot \beta_{2}-\csc \beta_{2}\right]$ & the boundary between regions $T_{1}$ and $R_{2}^{T}$ \\
$y=0$ & $\alpha_{2}=X\left(\cot \beta_{2} \csc \beta_{2}+\ln \left[\cot \beta_{2}-\csc \beta_{2}\right]\right)$ & the bottom of region $R_{2}^{T}$ \\
$y=(L-x) \sqrt{X /(L-X)}$ & $\beta_{2}=\theta_{0}$ & the top tie of region $R_{2}^{T}$ \\
$(X, 0)$ & $(0,-\pi / 2)$ & the bottom left corner of region $R_{2}^{T}$ \\
$(X, \sqrt{X(L-X)})$ & $\left(X \ln \left[\cot \theta_{0}-\csc \theta_{0}\right], \theta_{0}\right)$ & the top left corner of region $R_{2}^{T}$ \\
$(L, 0)$ & $\left(X\left(\cot \theta_{0} \csc \theta_{0}+\ln \left[\cot \theta_{0}-\csc \theta_{0}\right]\right), \theta_{0}\right)$ & the right corner of region $R_{2}^{T}$ \\
\hline
\end{tabular}


Equation (20) $)_{1}$ implies that $u_{2}=\ell \sigma\left(\alpha_{2} / \sigma_{T}+G\left(\beta_{2}\right)\right)$, with $G\left(\beta_{2}\right)$ chosen to ensure the continuity along the line $x=X$. Since $\beta_{1}$ and $\beta_{2}$ denote the same angle, the continuity with circumferential displacements requires $\left.u_{2}\right|_{\beta_{2}=\beta_{1}}=$ $-\left.v_{1}\right|_{\beta_{1}=\beta_{2}}$, so that a reference to $(11)_{2}$ provides the full definition

$u_{2}=\ell \sigma\left(\alpha_{2} / \sigma_{T}+G\left(\beta_{2}\right)\right)$,

where

$G\left(\beta_{2}\right)=X\left(\frac{\beta_{2}}{\sigma_{*}} \csc \beta_{2}-\frac{1}{\sigma_{T}} \ln \left[\cot \beta_{2}-\csc \beta_{2}\right]\right)$.

The rotation is fixed along $\alpha_{2}$-lines within $R_{2}^{T}$; therefore, the continuity of rotation along $x=X$ and (12) gives $\omega_{2}=$ $\ell \sigma \beta_{2} / \sigma_{*}$. This enables us to compute $v_{2}$ directly from $(20)_{2}$, with the result

$v_{2}=\ell \sigma\left(\frac{X \csc \beta_{2}}{\sigma_{C}}-\frac{\beta_{2}}{\sigma_{*}}\left(\alpha_{2}-X \ln \left[\cot \beta_{2}-\csc \beta_{2}\right]\right)\right)$.

The substitution of displacement (24) into (21) 1 again gives $\omega_{2}=\ell \sigma \beta_{2} / \sigma_{*}$, as it should. By substituting the value for $\alpha_{2}$ associated with $x=X$ from Table 1 into (24), it is also possible to verify that $\left.v_{2}\right|_{\beta_{2}=\beta_{1}}=\left.u_{1}\right|_{\beta_{1}=\beta_{2}}$. The remaining equation $(21)_{2}$ provides a direct means of computing the strain along $\beta_{2}$-lines, which is found to be

$\varepsilon_{2}^{\prime \prime}=\frac{2 \ell \sigma}{\sigma_{*}}\left(1-\frac{X \cot \beta_{2} \csc \beta_{2}}{\alpha_{2}+F\left(\beta_{2}\right)}\right)-\frac{\ell \sigma}{\sigma_{C}}$.

For the field within $R_{2}^{T}$ to satisfy the Michell criteria (5), we must ensure that $-\ell \sigma / \sigma_{C} \leqslant \varepsilon_{2}^{\prime \prime} \leqslant \ell \sigma / \sigma_{T}$. It is worth reminding ourselves that the denominator within (25) is an equation of the evolute. It can only vanish in a single point of region $R_{2}^{T}$, where the evolute touches the bottom left corner, see (14). However, due to the cancellation of terms, one has everywhere along the bottom boundary of $R_{2}^{T}$ :

$\left.\varepsilon_{2}^{\prime \prime}\right|_{y=0}=\ell \sigma / \sigma_{T}$, see (25) and Table 1. Simultaneously, everywhere along the boundary between regions $T_{1}$ and $R_{2}^{T}$,

$\left.\varepsilon_{2}^{\prime \prime}\right|_{x=L / 2}=-\ell \sigma / \sigma_{C}$.

Keeping in mind that, for every fixed $\beta_{2}, \varepsilon_{2}^{\prime \prime}$ is a monotonously increasing function of $\alpha_{2}$, see (25), that changes from $-\ell \sigma / \sigma_{C}$ at $x=L / 2$ to $\ell \sigma / \sigma_{T}$ at $y=0$, we come to the sought-for conclusion that $R_{2}^{T}$ is a valid Michell region of type $R^{T}$, see (5).

Having constructed a consistent strain field for a single half-span, we have not yet solved the original problem, featuring an infinite sequence of equally-spaced level supports. A full span of length $2 L$ can be obtained by reflecting the constructed fields with respect to $O y$. In addition, we can use (22), (24) and Table 1 to write $u_{2}^{x}$, the horizontal component of displacement along $y=0$, in the form

$$
\begin{aligned}
\left.u_{2}^{x}\right|_{y=0} & \left.\equiv u_{2}\right|_{y=0} \sin \phi_{2}+\left.v_{2}\right|_{y=0} \cos \phi_{2} \\
& =\ell \sigma\left(\frac{\cot ^{2} \beta_{2}}{\sigma_{T}}-\frac{1}{\sigma_{C}}\right) X .
\end{aligned}
$$

We intended to produce a symmetric structure, which can be reflected about the vertical $x=L$. This can only be done if the horizontal displacement $\left.u_{2}^{x}\right|_{y=0}$ vanishes at $x=L$, i.e. at $\beta_{2}=\theta_{0}$. Clearly, this happens when

$\theta_{0}=-\arctan \sqrt{\frac{\sigma_{C}}{\sigma_{T}}}$,

which, due to relation (10), is equivalent to

$X=\frac{\sigma_{C}}{\sigma_{C}+\sigma_{T}} L$.

Remarkably, condition (29) is also equivalent to the optimality condition obtained for the parabolic funicular loaded by a transmissible, uniformly distributed load, see Wang and Rozvany (1983) and Darwich et al. (2010). We have now identified the value of $X$ for which it is possible to produce a structure that, via a series of simple reflections and translations, is replicated across an infinite sequence of level pinned supports placed $2 L$ apart along $O x$. A depiction of a
Fig. 3 A single span of the constructed optimum structure in the case of equal allowable stresses

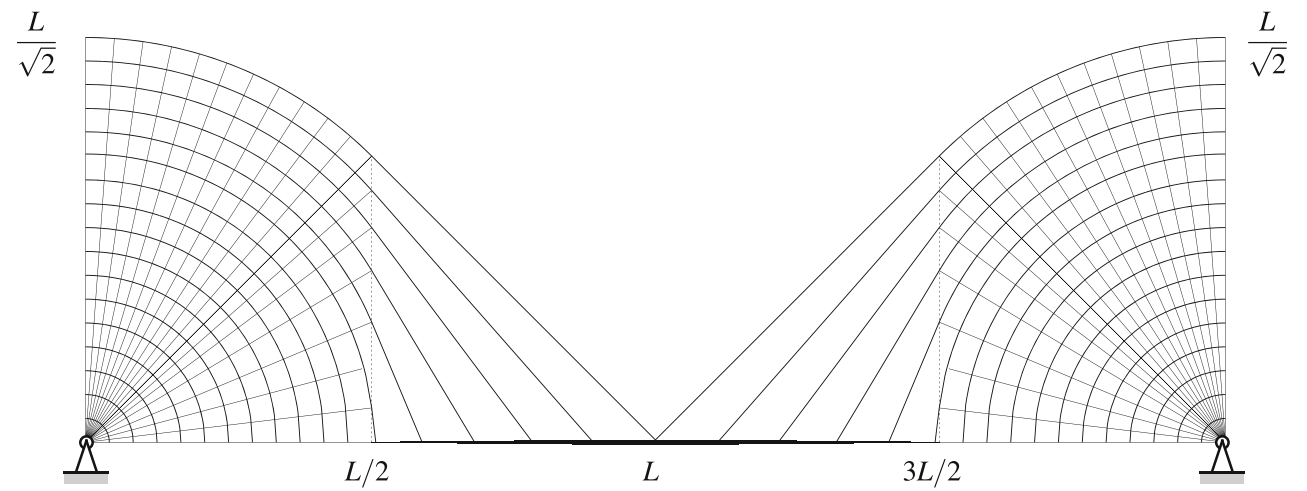


single span of such a structure for the important case when $\sigma_{C}=\sigma_{T}$ is presented in Fig. 3 .

It has already been mentioned that regions of type $T$, i.e. the regions that satisfy the Michell criteria and conditions (1), are often perceived to be synonymous with all Michell structures. Since these regions feature systems of mutually orthogonal members, the requirement of member orthogonality is often presumed for general Michell structures. This requirement is, evidently, violated at the bottom boundary of region $R_{2}^{T}$, where ties join a concentrated tensile member. Interestingly, Rozvany (1997) presents several examples showing how the orthogonality requirement can be relaxed along boundaries between $R^{C}$ and $R^{T}$ regions. The situation is simpler in our case. Since both principal strains become equal to $\ell \sigma / \sigma_{T}$ at the bottom boundary, see (26), one can interpret the concentrated member at the bottom of $R_{2}^{T}$ as a degenerate $S_{3}^{T}$ region (3), within which the orthogonality requirements do not hold.

Note that Cartesian point $(X, 0)$ is a singular point of the strain field. Within region $T_{1}$ the horizontal component of strain at $y=0$ is always compressive, i.e. $\varepsilon_{1}^{\prime}=-\ell \sigma / \sigma_{C}$. In particular, this is true at the boundary with $R_{2}^{T}$, if one approaches $(X, 0)$ along the vertical $x=X$, see (27). At the same time, if one approaches $(X, 0)$ along the involute $\alpha_{2}=0$ in $R_{2}^{T}$, i.e. takes the limit $\beta_{2} \rightarrow-\pi / 2$ of (25) with $\alpha_{2}=0$ fixed, or if one approaches $(X, 0)$ along the bottom of $R_{2}^{T}$, one obtains (26), i.e. the maximum allowable tensile strain. This singularity does not affect the continuity of the displacement or stress fields.

The virtual displacement field constructed in this section does not cover the space above the structure. This means that the described structure is guaranteed to be optimal only in the domain coinciding with the structure itself. However, our numerical investigation, which will be described in Section 6, strongly suggests that the described Michell structure is globally optimal for the upper half plane as long as $\sigma_{C} \leqslant \sigma_{T}$.

\section{The volume of the structure}

The volume of a single span of the proposed structure can be computed by calculating the work done by the external loads and dividing it by $\ell \sigma$. The work done $\mathscr{W}_{I}$ by the distributed load acting along $-X \leqslant x \leqslant X$ can be found with the help of expressions (9) and (30):

$\mathscr{W}_{I}=\ell \sigma \frac{\pi}{2} \frac{w X^{2}}{\sigma_{*}}=\ell \sigma \frac{\pi}{4} \frac{\sigma_{C}}{\sigma_{T}} \frac{w L^{2}}{\sigma}$.

In order to determine the work done $\mathscr{W}_{I I}$ by the distributed load acting along $X \leqslant|x| \leqslant L$, one needs to find the ver- tical displacement $u_{2}^{y}$ along the bottom boundary of $R_{2}^{T}$. Using (22), (24) and Table 1, we obtain

$$
\begin{aligned}
\left.u_{2}^{y}\right|_{y=0} & \equiv-\left.u_{2}\right|_{y=0} \cos \phi_{2}+\left.v_{2}\right|_{y=0} \sin \phi_{2} \\
& =\ell \sigma\left(\beta_{2} \csc ^{2} \beta_{2}+\cot \beta_{2}\right) X / \sigma_{*} .
\end{aligned}
$$

Using (32), the second work integral is computed as

$$
\begin{aligned}
\mathscr{W}_{I I} & =\left.2 \int_{X}^{L} w u_{2}^{y}\right|_{y=0} \mathrm{~d} x=4 w X \int_{-\pi / 2}^{\theta_{0}}-\left.u_{2}^{y}\right|_{y=0} \frac{\cos \beta_{2}}{\sin ^{3} \beta_{2}} \mathrm{~d} \beta_{2} \\
& =\ell \sigma\left(\frac{3 \sigma_{C}+5 \sigma_{T}}{6 \sqrt{\sigma_{C} \sigma_{T}}}-\frac{2 \sigma^{2} \theta_{0}}{\sigma_{C} \sigma_{T}}-\frac{\pi}{4} \frac{\sigma_{C}}{\sigma_{T}}\right) \frac{w L^{2}}{\sigma} .
\end{aligned}
$$

Clearly, $W_{\min }=\left(\mathscr{W}_{I}+\mathscr{W}_{I I}\right) / \ell \sigma$, hence

$$
\begin{aligned}
W_{\min } & =\left(\frac{3 \sigma_{C}+5 \sigma_{T}}{6 \sqrt{\sigma_{C} \sigma_{T}}}-\frac{2 \sigma^{2} \theta_{0}}{\sigma_{C} \sigma_{T}}\right) \frac{w L^{2}}{\sigma} \\
& =\left(\frac{3 \Delta_{\sigma}+5}{6 \sqrt{\Delta_{\sigma}}}+\frac{\left(1+\Delta_{\sigma}\right)^{2}}{2 \Delta_{\sigma}} \arctan \sqrt{\Delta_{\sigma}}\right) \frac{w L^{2}}{\sigma},
\end{aligned}
$$

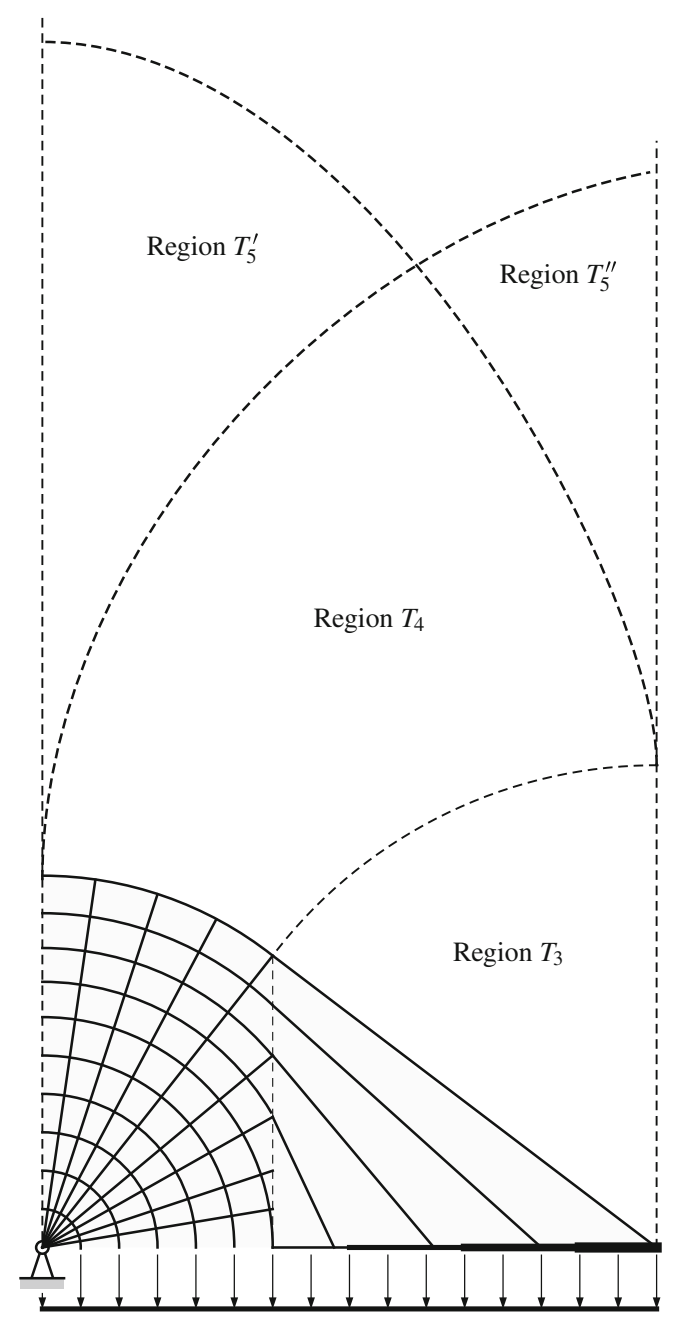

Fig. 4 The conjectured configuration of the virtual displacement field for the upper half-plane 
where $\Delta_{\sigma}=\sigma_{C} / \sigma_{T} \leqslant 1$. In the particularly important case of equal allowable stresses, the volume of a single span of the described structure is given by

$W_{\min }=\left(\frac{4}{3}+\frac{\pi}{2}\right) \frac{w L^{2}}{\sigma} \approx 2.90413 \frac{w L^{2}}{\sigma}$,

which is $11.0 \%$ lower than the volume of a simple parabolic arch with vertical hangers, and $7.86 \%$ lower than that of the classical solution obtained by Hemp (1974), which is known to be sub-optimal, see also Chan (1975).

\section{Global optimality}

Although we have now obtained a continuous virtual displacement field that satisfies all of our kinematic and static requirements, this does not constitute a proof of global optimality. A rigorous proof requires construction of a continuous virtual displacement field that covers the entire half-plane.

The derivation of such a field is beyond the scope of the present paper. However, we believe that geometry of the described Michell structure strongly suggests a likely configuration of the virtual displacement field above the structure, see the sketch in Fig. 4. In the proposed configuration, an additional region $T_{3}$ of lines and circles is placed above the region of inclined members $R_{2}^{T}$. Then the upper boundaries of regions $T_{1}$ and $T_{3}$ form two orthogonal circular arcs, which are sufficient to describe an additional fully strained region $T_{4}$, which will be largely identical to the cantilevers constructed by Chan (1962). Complementary to region $T_{4}$, there will need to be two additional regions $T_{5}^{\prime}$ and $T_{5}^{\prime \prime}$, sim-

Table 2 Numerical vs. analytical volumes, $V\left(\times w L^{2} / \sigma_{C}\right)$

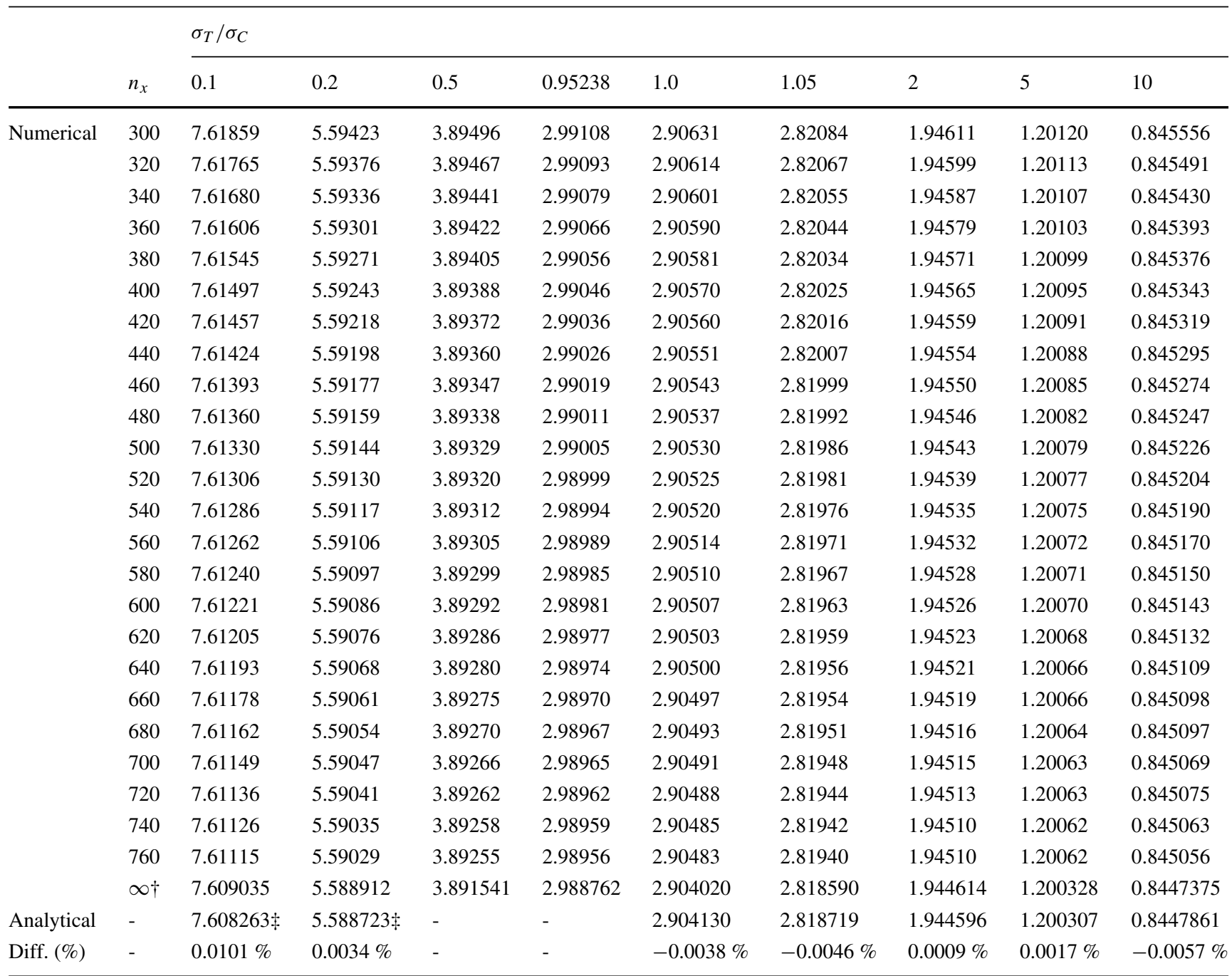

$\dagger$ Obtained by extrapolation, using a power-law extrapolation scheme as used by Darwich et al. (2010)

₹Analytical solution for arch with vertical hangers, after (Pichugin et al. 2012) 
ilar to, but not identical to the fields constructed by Chan (1967) (H. S. Y. Chan's solutions assume that the vertical boundaries of the domain are fixed, whereas in our case one must enforce the conditions for reflective symmetry). One can then construct further extensions of the field above, by adding further fully strained regions akin to the approach taken for Michell cantilevers by Lewiński et al. (1994), Graczykowski and Lewiński (2006a), Graczykowski and Lewiński (2006b), and Graczykowski and Lewiński (2007).

\section{Numerical solutions}

In order to verify the optimality of the structure described in previous sections, numerical solutions have been obtained using an efficient numerical layout optimization procedure by Gilbert and Tyas (2003). This procedure was also used to provide compelling numerical evidence that the parabolic arch is not an optimal structure to transfer a uniformly distributed transmissible load to two pinned supports
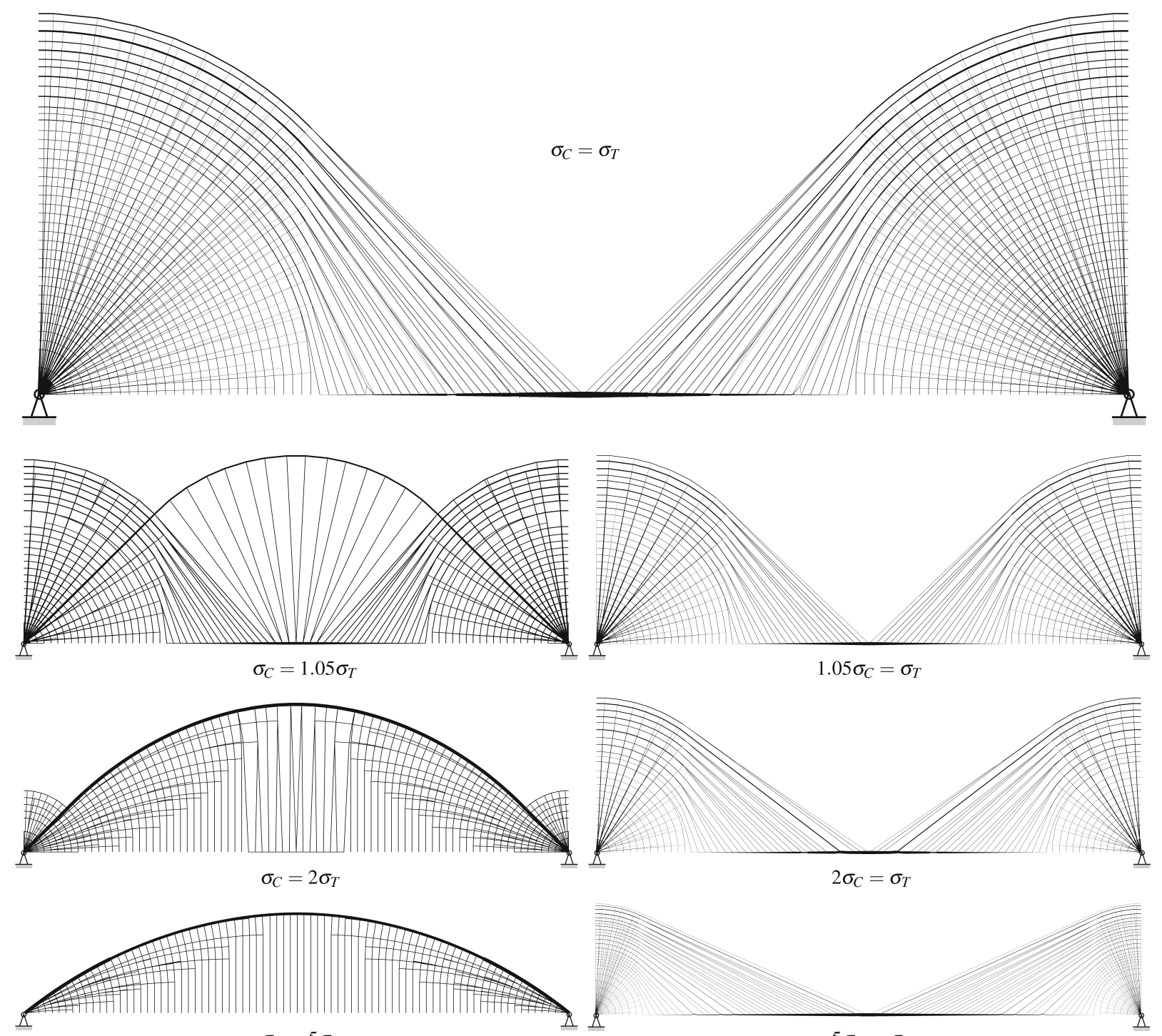

$$
\sigma_{C}=5 \sigma_{T}
$$
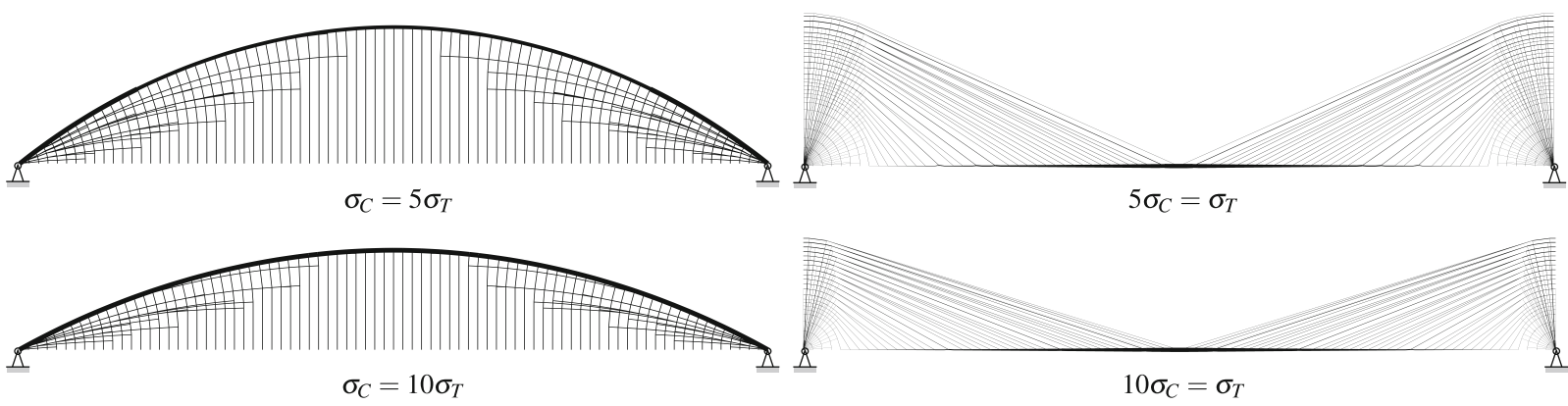

Fig. 5 Selected rationalized numerical solutions that illustrate the effects of varying the ratio of allowable stresses 
(Darwich et al. 2010), prior to formal proof of this being obtained by Tyas et al. (2011).

Detailed numerical results are shown in Table 2 for various ratios of the maximum allowable tensile and compressive stresses, $\sigma_{T}$ and $\sigma_{C}$, modelling half the problem domain (due to symmetry) with increasingly fine nodal resolutions. The largest model run, for the $\sigma_{C}=\sigma_{T}$ case, contained 10,591,164,111 potential members (i.e. over 10 billion potential members; see supplementary electronic material). Extrapolated volumes were also obtained using the power law extrapolation scheme described in Darwich et al. (2010). More specifically, models comprising $n_{x}=300,320, \ldots, 760$ divisions were used to provide source data, where $n_{x}$ is the number of nodal divisions across the full span. A weighted nonlinear leastsquares approach was used to find best-fit coefficient values for use in the extrapolation, with the weighting factor taken as $n_{x}$, to increase the influence of fine resolution solutions.

It is clear from Table 2 that the extrapolated numerical solutions agree extremely well with the analytical solutions presented in this paper for the $\sigma_{T} \geqslant \sigma_{C}$ cases, suggesting that the analytical solutions presented are likely to be globally optimal for the half plane. (In fact the very high levels of accuracy that can now be obtained using modern numerical methods and computational resources would seem to indicate that the latter will increasingly provide a pragmatic means of confirming global optimality.)

Fine resolution numerical layout optimization solutions are often complex in form, particularly when uniform loads are involved (since equilibrium requirements dictate that each loaded node must be connected by one or more members). Consequently, for sake of clarity, Fig. 5 shows relatively coarse resolution numerical solutions. These have been rendered even clearer via application of a geometry optimization post-processing step, which involves rationalizing the solution by adjusting the positions of nodes (He and Gilbert 2015); full details of the numerical solutions are included in the electronic supplementary information.

It is evident from Fig. 5 that the structure obtained when $\sigma_{C}=\sigma_{T}$ (shown at the top of the figure) displays a remarkable similarity to the analytical solution shown in Fig. 3. Also, when $\sigma_{T}>\sigma_{C}$, the numerical results are very similar to the layout postulated in Fig. 2, with the parameter $X$, which defines the location at which half-wheel fields are replaced by systems of straight tension members decreasing according to the ratio of $\sigma_{C}$ and $\sigma_{T}$, as predicted by (30). However, when $\sigma_{T}<\sigma_{C}$, a different structure emerges. In this case an arch compression rib with inclined tensions hangers develops over the central section of the span, with a Hencky-net fan emerging from the Michell wheel-like section closer to the support. Interestingly, the numerical solutions for unequal allowable stresses indicate that our solution, although seemingly unusual, is closely related to two well known classes of structure, widely used in engineering practice. For example, the left hand side of Fig. 5 presents structures dominated by compression $\left(\sigma_{C}>\sigma_{T}\right)$. As $\sigma_{C} / \sigma_{T}$ increases, the fans around the supports shrink in size; at $\sigma_{C} / \sigma_{T} \approx 3.70$, this fan vanishes entirely and the structural form of the overall structure tends towards a simple arch with vertical hangers, with the numerical solutions tending towards the analytical solutions obtained by Pichugin et al. (2012). Conversely, in the case of structures dominated by tension $\left(\sigma_{C}<\sigma_{T}\right)$, shown on the right hand side of Fig. 5, the solutions metamorphose towards a cable-stayed bridge-like structure, with the fans shrinking to become stocky, near-vertical, towers. This remarkable and entirely unexpected result suggests that arch and cablestayed bridge structures lie at opposite ends of a continuous spectrum of optimal structural forms.

\section{Conclusions}

Details of a new half-plane Michell structure capable of carrying a uniformly distributed load of infinite horizontal extent over a series of equally-spaced pinned supports have been presented. Although formal proof of optimality of the structure has not yet been demonstrated, the proposed analytical solution is supported by results from high-resolution numerical simulations. Numerical solutions also suggest the existence of a wider family of related, simple, and practically relevant structures, which range in form from an arch with vertical hangers to a cable-stayed bridge, depending on the specified ratio of limiting compressive to tensile stress.

\section{References}

Chan ASL (1962) The design of Michell optimum structures. Reports \& Memoranda No. 3303. Her Majesty's Stationery Office, London Chan HSY (1967) Half-plane slip-line fields and Michell structures. Q J Mech Appl Math 20(4):453-469. doi:10.1093/qjmam/20.4.453

Chan HSY (1975) Symmetric plane frameworks of least weight. In: Sawczuk A, Mŕoz Z (eds) Optimization in Structural Design. Springer, Berlin, pp 313-326

Darwich W, Gilbert M, Tyas A (2010) Optimum structure to carry a uniform load between pinned supports. Struct Multidiscip Optim 42(1):33-42. doi:10.1007/s00158-009-0467-0

Gilbert M, Tyas A (2003) Layout optimization of largescale pin-jointed frames. Eng Comput 20(8):1044-1064. doi:10.1108/02644400310503017

Graczykowski C, Lewiński T (2006a) Michell cantilevers constructed within trapezoidal domains-Part I: geometry of Hencky nets. Struct Multidiscip Optim 32(5):347-368. doi:10.1007/s00158-005-0599-9 
Graczykowski C, Lewiński T (2006b) Michell cantilevers constructed within trapezoidal domains-Part II: virtual displacement fields. Struct Multidiscip Optim 32(6):463-471. doi:10.1007/s00158-005-0600-7

Graczykowski C, Lewiński T (2007) Michell cantilevers constructed within trapezoidal domains-Part III: force fields. Struct Multidiscip Optim 33(1):1-19. doi:10.1007/s00158-005-0601-6

He L, Gilbert M (2015) Rationalization of trusses generated via layout optimizatiom. Struct Multidiscip Optim. In press. doi:10.1007/s00158-015-1260-x

Hemp WS (1973) Optimum Structures. Clarendon Press, Oxford

Hemp WS (1974) Michell framework for uniform load between fixed supports. Eng Optim 1(1):61-69. doi:10.1080/03052157408960577

Lewiński T, Zhou M, Rozvany GIN (1994) Extended exact solutions for least-weight truss layouts-Part I: cantilever with a horizontal axis of symmetry. Int J Mech Sci 36(5):375-398. doi:10.1016/0020-7403(94)90043-4

Michell AGM (1904) The limits of economy of material in frame-structures. Philos Mag 8(47):589-597. doi:10.1080/14786440409463229

Morse PM, Feshbach H (1953) Methods of theoretical physics, Part 1. McGraw-Hill Book Company, Inc., New York

Pichugin AV, Tyas A, Gilbert M (2011) Michell structure for a uniform load over multiple spans. In: Proceedings of the Ninth
World Congress on Structural and Multidisciplinary Optimization (WCSMO-9). Shizuoka, Japan

Pichugin AV, Tyas A, Gilbert M (2012) On the optimality of Hemp's arch with vertical hangers. Struct Multidiscip Optim 46(1):17-25. doi:10.1007/s00158-012-0769-5

Rozvany GIN (1996) Some shortcomings in Michell's truss theory. Struct Optim 12(4):244-250. doi:10.1007/BF01197364

Rozvany GIN (1997) Partial relaxation of the orthogonality requirement for classical Michell trusses. Struct Optim 13(4):271-274. doi:10.1007/BF01197457

Rozvany GIN (1998) Exact analytical solutions for some popular benchmark problems in topology optimization. Struct Optim 15(1):42-48. doi:10.1007/BF01197436

Rozvany GIN, Bendsøe MP, Kirsch U (1995) Layout optimization of structures. Appl Mech Rev 48(2):41-119. doi:10.1115/1.3005097

Sokół T, Lewiński T (2010) On the solution of the three forces problem and its application in optimal designing of a class of symmetric plane frameworks of least weight. Struct Multidiscip Optim 42(6):835-853. doi:10.1007/s00158-010-0556-0

Tyas A, Pichugin AV, Gilbert M (2011) Optimum structure to carry a uniform load between pinned supports: exact analytical solution. Proc R Soc A 467(2128):1101-1120. doi:10.1098/rspa.2010.0376

Wang CM, Rozvany GIN (1983) On plane Prager-structures-II. Nonparallel external loads and allowances for selfweight. Int J Mech Sci 25(7):529-541. doi:10.1016/0020-7403(83)90045-0 